\title{
Tolerating Stuck-at Fault and Variation in Resistive Edge Inference Engine via Weight Mapping
}

\author{
Yu Ma \\ School of Information Science and \\ Technology \\ ShanghaiTech University \\ Shanghai, China \\ mayu@shanghaitech.edu.cn
}

\author{
Linfeng Zheng \\ School of Information Science and \\ Technology \\ ShanghaiTech University \\ Shanghai, China \\ zhenglf@shanghaitech.edu.cn
}

\author{
Pingqiang Zhou \\ School of Information Science and \\ Technology \\ ShanghaiTech University \\ Shanghai, China \\ zhoupq@shanghaitech.edu.cn
}

\begin{abstract}
There is an increasing demand for running neural network inference on edge devices. Memristor crossbar array (MCA) based accelerators can be used to accelerate neural networks on edge devices. However, reliability issues in memristors, such as stuck-at faults (SAF) and variations, lead to weight deviation of neural networks and therefore have severe influence on inference accuracy. In this work, we focus on reliability issues for edge devices. We formulate the reliability problem as a $0-1$ programming problem, based on the analysis of sum weight variation (SWV). In order to solve the problem, we simplify the problem with an approximation - different columns have the same weights - based on our observation of the weight distribution. Then we propose an effective mapping method to solve the simplified problem. The experimental results show that our proposed method can recover $95 \%$ accuracy considering SAF defects and can increase by up to $60 \%$ accuracy in variation $\sigma=0.4$.
\end{abstract}

\section{CCS CONCEPTS}

- Hardware $\rightarrow$ Emerging technologies; • Computer systems organization $\rightarrow$ Reliability.

\section{KEYWORDS}

Memristor, Variation, Stuck-at fault, Neural networks, Edge computing

\section{ACM Reference Format:}

Yu Ma, Linfeng Zheng, and Pingqiang Zhou. 2021. Tolerating Stuck-at Fault and Variation in Resistive Edge Inference Engine via Weight Mapping. In Proceedings of the Great Lakes Symposium on VLSI 2021 (GLSVLSI '21), fune 22-25, 2021, Virtual Event, USA. ACM, New York, NY, USA, 6 pages. https://doi.org/10.1145/3453688.3461487

\section{INTRODUCTION}

Data generated by edge devices are substantially growing [1]. Thus, the demand for running neural networks inference on edge devices

Permission to make digital or hard copies of all or part of this work for personal or classroom use is granted without fee provided that copies are not made or distributed for profit or commercial advantage and that copies bear this notice and the full citation on the first page. Copyrights for components of this work owned by others than ACM must be honored. Abstracting with credit is permitted. To copy otherwise, or republish, to post on servers or to redistribute to lists, requires prior specific permission and/or a fee. Request permissions from permissions@acm.org.

GLSVLSI '21, fune 22-25, 2021, Virtual Event, USA

(C) 2021 Association for Computing Machinery.

ACM ISBN 978-1-4503-8393-6/21/06 . \$ \$15.00

https://doi.org/10.1145/3453688.3461487 is increasing. In the inference process of neural networks, the inputs and weights are multiplied and accumulated (MAC), which is the most expensive operation phase. In order to accelerate this process, memristor crossbar array (MCA) structure is suitable on edge devices [2]. It can execute MAC operations using Kirchhoff's law natively [3] by representing inputs and weights with voltages and conductances respectively.

There exist two methods to program the conductances of memristors. One is "Close-Loop on-Device" [4] which requires costly hardwares [5]. The other method, which is suitable for edge devices, trains networks off-line and then programs memristors with pre-calculated voltage magnitudes and pulse widths [6]. Because of the reliability issues (i.e. defects and variations) of memristors, the programmed conductances are not equal to the desired conductances which represent the weights of the given neural network. This leads to the accuracy degradation of neural networks [7].

There are two critical reliability issues of memristors: stuck-atfault (SAF) defect [8] and variation [5]. Some researchers propose permutation and remapping methods [5, 9-12]. This process needs very large amount of computation which is intolerable for edge devices. Other researchers propose retraining methods [7, 9], which is computational expensive for computing platforms [8]. Besides, Some other researchers propose quantization methods to enhance the variation tolerant capability [13], the results of which show that the method can only tolerate a small variation.

All previous works don't consider the limitation of crossbar size. Although the crossbar size can be large ideally, the size is always limited in reality [14] because of power and reliability issues [15]. Therefore, multiple crossbars are integrated together using a shared interconnect, for example Networks on Chips (NoCs) (see Figure 1 (c)). Large matrices are always separated into pieces and then mapped to several MCAs. Then the peripheral circuit combines the results of the MCAs together. In this paper, we propose a mapping method to tolerate both SAF and variation of memristors considering size limitation and the characteristics of edge devices stringent resources. The contributions of this paper are summarized as below:

- We analyze MCA-based edge devices and point out the suitable computing method - representing one weight with one memristor because of the limited resources and vector-vector multiplication to eliminate the sneak path problem.

- According to the above-mentioned circumstance, we analyze the weight deviation caused by SAF and variation and formulate the optimization problem as a $0-1$ programming problem. 


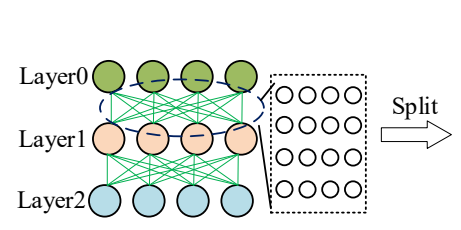

(a)

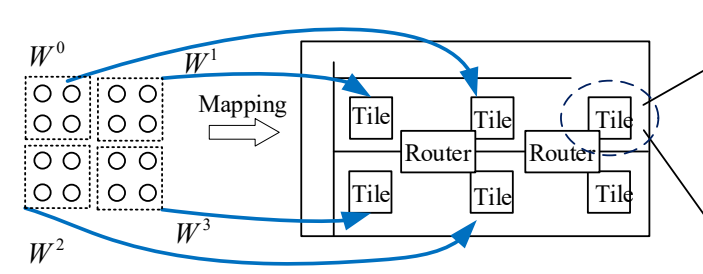

(b)

(c)



(d)

Figure 1: Architecture of MCA Based Devices. (a) A neural network, (b) Weight splitting, (c) Weight mapping. (d) Architecture of MCA based accelerators.

- Since the 0-1 programming is hard for edge devices to find a good solution, we simplify the problem with an observation - different columns have similar values in neural networks. Then we propose an optimal mapping method for the simplified problem and modify the computing pipeline of MCAs.

- We evaluate our proposed method with two feed forward neural networks on the MNIST dataset. The experimental results show that the proposed method can increase the reliability of MCAs for edge devices. The inference accuracy can be maintained even if the SAF rate reaches $30 \%$. Our proposed method can tolerate variation $\sigma=0.4$.

The rest of this paper is organized as follows. Section 2 introduces MCA-based neural network inference according to characteristics of edge devices and the model of SAF defect and variation. Section 3 formulates the problem and introduce our proposed method. At last, Section 4 shows our experimental results, followed by conclusion in Section 5.

\section{BACKGROUND}

Edge devices, which are used to execute real time tasks such as translation and objective recognition, have stringent computing resources and tight power budget [16]. Memristor crossbar array (MCA) based accelerators can be used in edge devices.

\subsection{Memristor Crossbar Based Computing}

In order to accelerate a given neural network, the inference process with MCAs is shown in Figure 1.

- Firstly, the neural network is trained off-line and Figure 1 (a) shows a three-layer network and the weight matrix of the first-layer as an example. In this work, the row and column numbers of the weight matrix are the input and output sizes respectively.

- Before executing the inference, the weights of neural networks need to be mapped to MCAs. Because of the limited size of MCAs [17], the weight matrix is firstly split to pieces (see Figure 1 (b)).

- Then each piece of weights are mapped to MCAs (see Figure $1(\mathrm{c}))$. In this process, conductances of memristors are programmed according to weights. Although one weight can be represented by multiple memristors to increase the robustness of computing [8], because of the limited resources in edge devices, we consider the case that one memristor represents one weight (see Figure $2(a)$ ) in our work. Each gate line (see Figure 2 (b)) controls transistors of a certain column. In the programming phase, each memristor is programmed to a certain conductance according to the weight. In the inference phase, transistors of gate lines are open and current is able to flow through memristors.

Supposing we represent each weight with one memristor, conductance of the memristor is programmed linearly according to weights (see Figure 2 (b)) which can be expressed as Equation (1) [18]:

$$
G=\frac{G_{\max }-G_{\min }}{W_{\max }-W_{\min }}\left(W-W_{\min }\right)+G_{\min }
$$

where $G_{\min }$ and $G_{\max }$ are the minimum and maximum conductances of the memristor, $W_{\min }$ and $W_{\max }$ are the minimum and maximum weights.

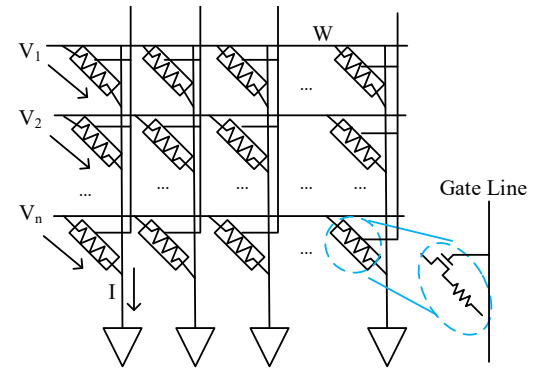

(a)



(c)
Figure 2: MAC operation. (a) Schematic of 1T1R array [19] and MAC operation. (b) 1T1R cell. (c) Linear one-to-one mapping.

After mapping weights to MCAs, in the inference phase, the inputs are fed to input registers (IRs) of MCAs firstly. For a certain layer, in each cycle, each bit of input is fed to the crossbars and every column in crossbars executes MAC operation in parallel according to Kirchhoff's law, $I=\vec{G} \cdot \vec{V}$ (see Figure 2 (a)). The result of each cycle is shifted and added $(\mathrm{S}+\mathrm{A})$ (see Figure $1(\mathrm{~d})$ ). After all bits are computed, the results of each MCA are combined to get the result of the layer. After activation, the result is transferred to the next layer. After the last layer is processed, the inference result is generated.

\subsection{Defect and Variation Modeling}

Ideally, the conductance of a memristor can be programmed according to a given weight (see Equation (1)), however, there are defects and variations of memristors. In this work, we consider two reliability issues related to memristors - SAF defect and variation.

2.2.1 Stuck at Fault. SAF defect is the most critical reliability issue in memristor crossbar [8]. Assuming that we want to program the 
conductance of one memristor to $G$, the conductance state is not able to be changed and is stuck at high (stuck-on) or low (stuckoff), if the memristor suffers from SAF defect. Considering this, the programmed conductance can be expressed as Equation (2):

$$
G^{\prime}=\left\{\begin{array}{l}
G_{\min }, \text { stuck-off } \\
G, \text { no defect } \\
G_{\max }, \text { stuck-on }
\end{array}\right.
$$

where $G^{\prime}$ is the conductance we program in practice. If the memristor suffers from this issue, the weight which is programmed to this memristor is fixed because the conductance represents the weight. In this case, the accuracy of the given neural network degrades [7]

2.2.2 Variation. In addition to SAF defect, variation also has a huge influence on inference accuracy of MCA-based neural networks [5, 9]. As conductance is programmed with pre-calculated voltage magnitude and pulse width, the conductance we program in practice follows a lognormal distribution [20] which can be expressed as Equation (3):

$$
G^{\prime}=G \cdot e^{\theta}
$$

where $G^{\prime}$ is the programmed conductance in practice, $G$ is the conductance we want to program, and $\theta \sim N\left(0, \sigma^{2}\right)$ is the variation distribution.

2.2.3 SWV Metric. Because of the defect and variations of memristors, the effective weights represented by the memristors are not accurate. In order to evaluate the programming error, sum weight variation (SWV) [5] is the metric to calculate the difference between weight $(W)$ and effective weight $\left(W^{e f f}\right)$ :

$$
S W V=\sum_{i}\left|w_{i}^{e f f}-w_{i}\right|
$$

where $W_{i}$ is the $i$-th weight of weight matrix.

\section{PROPOSED METHOD}

In this section, we optimize SWV metric by assigning weights to different MCAs in the mapping process. Generally, computing with all columns in parallel leads to high power and more computing resources. If all columns are computed together, each layer can be computed in $10^{3} \mathrm{~ns}$ [21], which is overpowered for edge devices. Secondly, the power consumption is too large for an edge device. Further more, sneak path [22] is also an import reliability issue. Considering these three aspects, we apply column by column computing method in our work (this satisfies the performance requirement which will be introduced in Section 3). We further provide a new direction to improve the reliability of MCA-based neural network inference for edge devices.

\subsection{Problem Formulation}

In system level, digital results from MCAs are gathered and processed before producing the output of one layer because the weight matrices are split to pieces. As a result, we consider the piece of weight matrix which is mapped to the $k$-th MCA, i.e.:

$$
G=\frac{G_{\max }-G_{\min }}{W_{\max }^{k}-W_{\min }^{k}}\left(W-W_{\min }^{k}\right)+G_{\min }
$$

where $W_{\min }^{k}$ and $W_{\max }^{k}$ are the minimum and maximum weights in the $k$-th MCA. As there are SAF defect and variation of memristors in MCAs, the weights which are programmed to memristors are not accurate. We analyze the weight deviation in the programming phase. If we program one memristor to conductance $G$, we can represent the conductance deviation as $\Delta G\left(\Delta G=G^{\prime}-G\right)$ because of the SAF defect and variation. According to Equation (5), the effective weight $W^{\text {eff }}$ can be expressed as:

$$
W^{e f f}=W_{\min }^{k}+\frac{W_{\max }^{k}-W_{\min }^{k}}{G_{\max }-G_{\min }}\left(G+\Delta G-G_{\min }\right)
$$

As a result, considering one certain weight, the error between the changed weight and the initial weight can be express as:

$$
\left|W^{e f f}-W\right|=\frac{W_{\max }^{k}-W_{\min }^{k}}{G_{\max }-G_{\min }} \cdot|\Delta G|
$$

Then, considering that the weight matrix is split to several IMAs, the SWV of one weight matrix is the sum of SWVs of every IMA which can be expressed as:

$$
\begin{aligned}
S W V & =\sum_{k \in M C A s} S W V_{k} \\
& =\sum_{k \in M C A s} \sum_{i \in M C A_{k}} \frac{W_{\max }^{k}-W_{\min }^{k}}{G_{\max }-G_{\min }} \cdot\left|\Delta G_{k, i}\right|
\end{aligned}
$$

where $\Delta G_{k, i}$ is the conductance deviation at the $i$-th position in the $k$-th MCA. In Equation (8), $G_{\max }$ and $G_{\min }$ are constants and $\Delta G$ is subject to a distribution because of the SAF defect and variation. Therefore, we can minimize the sum of the $\left(W_{\max }-W_{\min }\right)$ of each MCA to minimize SWV:

$$
\min S W V \sim \min \sum_{k}\left(W_{\max }^{k}-W_{\min }^{k}\right)
$$

As a result, our objective can be minimizing the range of the weights which are mapped to each MCA while ensuring that MAC operation can be applied. We can formulate this problem as an $0-1$ programming problem as Equation (10) - (15).

$$
\begin{aligned}
\min _{\delta_{i, j, k}} & \sum_{k}\left(\max _{i, j}\left(\delta_{i, j, k} \cdot w_{i, j}\right)-\min _{i, j}\left(\delta_{i, j, k} \cdot w_{i, j}\right)\right) \\
\text { s.t. } & \delta_{i, j, k} \in\{0,1\}, \quad \forall i, j, k \\
& \sum_{k} \delta_{i, j, k}=1, \quad \forall i, j \\
& l_{k, j} \in\{0,1\} \quad \forall k, j \\
& \sum_{j} l_{k, j}=S, \quad \forall k \\
& \sum_{i} \delta_{i, j, k}=l_{k, j} \cdot S, \quad \forall j, k
\end{aligned}
$$

where $i=0,1, \cdots, R, j=0,1, \cdots, C, k=0,1, \cdots, K$, and $R$ and $C$ are numbers of rows and columns of one weight matrix respectively, $S$ is the size of MCA (assume MCA is square) and $K=\lceil R / S\rceil \times\lceil C / S\rceil$ is the number of MCAs to use, $w_{i, j}$ is the $i$-th row $j$-th column weight in the weight matrix, $l_{k, j}=1$ indicates that there are weights in $j$-th column mapped to the $k$-th MCA, $\delta_{i, j, k}$ is to record whether $w_{i, j}$ is mapped to the $k$-th MCA.

Equation (12) ensures that every weight is mapped and can be only mapped to one MCA, Equation (14) ensures that $S$ columns 
are mapped to each MCA and Equation (15) ensures that $S$ items in $j$-th column are mapped to $k$-th MCA. The Equation (14) and (15) ensure that we can execute MAC operation in MCAs, i.e. the weights, that are mapped to the same column in one MCA, are in the same column of weight matrix.

\subsection{Proposed Solution}

We formulate the weight mapping problem in previous section. In this section, we propose our method for solving this problem. As $0-1$ programming problem is a difficult optimization problem and it's inappropriate for edge devices to solve. As a result, we propose a simple approximate solution for this problem.

3.2.1 Problem Simplification. In order to simplify the problem, we analyze the weight distribution of neural networks. We analyze one layer (size $784 \times 1000$ ) of a well-trained network as an example. We sort weights in each column and then compare the difference of weights among the sorted columns. Then we calculate the normalized average difference (NAD) between two columns, i.e. average difference divided by the range of the two columns. We find that NAD of the most different two columns is smaller less than $10 \%$. As for the two most similar columns, the NAD is smaller than $0.5 \%$, the distributions of which are shown in Figure 3. To summarize, in neural networks, we observe that weight distributions in different columns are very similar. In order to simplify the programming problem (10), we approximately assume that different columns have the same weights. For example, as shown in Figure 4 (a), weights in every column are $\{1,2,3,4,5\}$.


Figure 3: Weight distribution of the two most similar columns.

3.2.2 Mapping Method. Our purpose is to map similar values to the same MCA with the assumption that different columns have the same weights. Our proposed mapping method is shown in Algorithm 1. Given a weight matrix with size $R \times C$ and MCA size $S$, our proposed method is to determine which weight should be mapped to which MCA. Firstly, we compute the number of MCAs we need to map the weight as $\lceil R / S\rceil \times\lceil C / S\rceil$ (line 1) and judge whether the weight matrix can fill all the MCAs assigned for it (line 2). If not, we add rows or columns to the end of weight matrix to fill all MCAs (line 3). Then we sort the values of weight matrix by columns and store the sorting index matrix (line 6). At last, we map the sorted matrix to MCAs in order (line 8) and return the mapping label $\delta$.



For example, Figure 4 (a) shows a $5 \times 5$ matrix we need to map and the size of MCAs is 3. Initially, the weight matrix is chaotic (see Figure $4(\mathrm{a})$ ). Then we need $\lceil 5 / 3\rceil \times\lceil 5 / 3\rceil=4 \mathrm{MCAs}$ and then insert one dummy column and one dummy row for this matrix (see Figure 4 (b)). Thus, MCA0 - MCA3 are assigned for this weight matrix. After that, we sort the weights by columns (see Figure 4 (c)). At last, we assign the weight matrix to MCAs as shown in Figure 4 (d). We can get the mapping label $\delta$. For example, the location $(0,0)$ is mapped to MCA0 and location $(4,4)$ is mapped to MCA3, therefore $\delta_{0,0,0}=1$ and $\delta_{4,4,3}=1$.

\begin{tabular}{|c|c|c|c|c|c|c|c|}
\hline \multirow[t]{2}{*}{$\begin{array}{ccccc}1 & 2 & 3 & 4 & 5 \\
2 & 3 & 4 & 5 & 1 \\
3 & 4 & 5 & 1 & 2 \\
4 & 5 & 1 & 2 & 3 \\
5 & 1 & 2 & 3 & 4\end{array}$} & insert & \begin{tabular}{|llllll}
1 & 2 & 3 & 4 & 5 & $\mathrm{x}$ \\
2 & 3 & 4 & 5 & 1 & $\mathrm{x}$ \\
3 & 4 & 5 & 1 & 2 & $\mathrm{x}$ \\
4 & 5 & 1 & 2 & 3 & $\mathrm{x}$ \\
5 & 1 & 2 & 3 & 4 & $\mathrm{x}$
\end{tabular} & sort & $\begin{array}{llllll}1 & 1 & 1 & 1 & 1 & x \\
2 & 2 & 2 & 2 & 2 & x \\
3 & 3 & 3 & 3 & 3 & x \\
4 & 4 & 4 & 4 & 4 & x \\
5 & 5 & 5 & 5 & 5 & x \\
\end{array}$ & $\begin{array}{l}\text { MCA0 } \\
\text { assign }\end{array}$ & 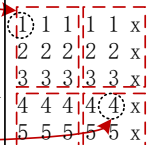 & \begin{tabular}{|l} 
MCA2 \\
1 \\
1
\end{tabular} \\
\hline & & $\begin{array}{lllllll}X & X & X & X & X & X \\
\end{array}$ & & $\begin{array}{llllll}X & x & x & x & x & x\end{array}$ & & 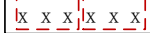 & i] $\mathrm{MC}$ \\
\hline
\end{tabular}

(a)

(b)

(c)

(d)

Figure 4: Mapping Method.

In our solution, all the weights are mapped only once, and thus, Equation (12) is satisfied. We don't exchange weights between two columns, and as a result, Equation (14) and Equation (15) are also satisfied. Besides, our proposed method only needs sorting operation which is very simple and therefore suitable for edge devices. Our proposed method is optimal for the simplified problem (because of the limited space, we don't prove it here). Thus, our proposed method can increase the robustness of the computing considering variation and SAF.

\subsection{Computing Pipeline}

As we compute with MCA column by column, we need to modify the computing pipeline from the conventional method which is introduced in Section 2. Input data are stored in the input registers (IRs). The required data are sent to IRs by routers cycle by cycle. In the first cycle, data are fed to IRs by routers. In the following cycles, the first column is computed by the MCA bit by bit. After that, the second column is computed and so on. For example, the input of one layer and the weight assignment are shown in Figure 5. Figure 5 (a) shows the input and Figure 5 (b) shows which data are 
fed to which MCA. The upper data are feed to MCA0 and MCA2, the lower data are fed to MCA1 and MCA3 according to our mapping label (generated in mapping phase) by routers in the first cycle. Assuming that the input data are 16-bit, in the $2^{\text {nd }}$ to $17^{\text {th }}$ cycle, the first columns of layers of the MCAs are computed bit by bit. In the next 16 cycles, the second columns are computed. After all the columns are computed, peripheral circuits gather the results of MCAs and transfer the activation to the next layer.

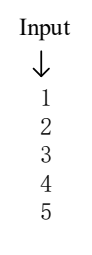

(a)

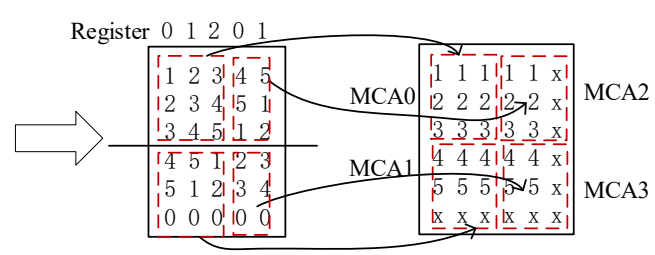

(b)

Figure 5: Computing Pipeline.

Without loss of generality, each cycle is set to 100ns [21], and therefore, each layer of neural networks can be computed within $10^{6} \mathrm{~ns}$ if size of MCA is 100 . Because of the pipeline design, this system can reach $10^{3}$ frames per second (FPS). Generally, real-time applications need about $10^{2}$ FPS computing power. Although our computing method causes decrement of the throughput, this method is still available for edge devices.

\section{EXPERIMENTAL RESULTS AND DISCUSSION}

In order to evaluate the effectiveness of our proposed highly reliable mapping method, we compare the neural network inference accuracy with our proposed method and without our proposed method considering SAF and variation. On the other hand, our proposed method introduces overhead to the accelerator. As a result, we also discuss the overhead of our proposed method after our experimental results. Following the practice of previous works [8, 9], we evaluate our proposed method with two feed-forward neural networks (see Table 1) on the MNIST dataset [23] and the minimum and maximum nominal resistance are set to $10 k$ and $1 M \Omega$ respectively. The SAF defects and variations are modeled with Equation (2) and (3) respectively. The size of MCAs are set to 64 [17]. The experiments are tested with the Monte-Carlo simulation method. OP and NO denote the accuracy with and without our proposed method respectively. We evaluate our proposed method in three aspects:

- SAF evaluation. We evaluate the effectiveness of our proposed method with different SAF defect rates.

- Variation evaluation. We evaluate the accuracy under different variations.

- Scalability. As our proposed method is sensitive to weight matrix and MCA size, we analyze the scalability of our proposed method.

Table 1: Properties of neural networks in experiments.

\begin{tabular}{|c|c|c|}
\hline Number of layers & Dimension & Ideal Accuracy \\
\hline 2 & $784 \times 10$ & $91.47 \%$ \\
\hline 3 & $784 \times 256,256 \times 10$ & $95.73 \%$ \\
\hline
\end{tabular}

\subsection{Results for SAF Defect}

We evaluate SAF effectiveness assuming that the defects are distributed randomly in each MCA and $81.6 \%$ of the defects are stuckon and $18.4 \%$ of the defects are stuck-off [7].

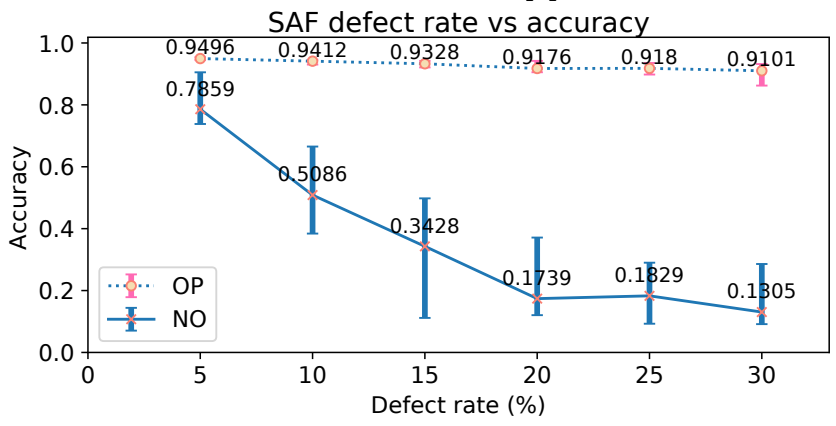

Figure 6: Accuracy vs SAF defect rate on the 3-layer network.

The accuracy results with different defect rates are shown in Figure 6. Without our proposed method, the performance of the network is influenced largely and accuracy degrades below $80 \%$ with only $5 \%$ defect rate. If the defect rate is increased to $20 \%$, the inference accuracy of the network is below $20 \%$.

With our proposed method, the inference accuracy is almost not influenced (maintaining about 95\% accuracy) with 5\% defect rate and can maintain a high accuracy $(91 \%)$ with even $30 \%$ defect rate.

Table 2: Comparison with other methods using the 3-layer neural network and $20 \%$ defect rate.

\begin{tabular}{|c|c|c|c|}
\hline Method & {$[8]$} & {$[7]$} & Ours \\
\hline Recovered accuracy & $30.1 \%$ & $95 \%$ & $95.9 \%$ \\
\hline
\end{tabular}

Besides, as shown in Table 2, we compare our proposed method with state of the art methods $[7,8]$ with $20 \%$ defect rate. The recovered accuracy is defined as accuracy normalized with the ideal classification accuracy of the neural network [8]. With stringent resources (one memristor represent one weight), our proposed method reaches $95.9 \%$ recovery accuracy. [8] can only get $30.1 \%$ while this method can get $97.6 \%$ recovered accuracy with multiple memristors representing one weight. Although [7] can also reach 95\% recovery accuracy, our proposed method doesn't need retraining process which is a very costly process for platforms [8]. As a result, our method is friendly for edge devices and can reach state of the art effectiveness.

\subsection{Results for Variation}

In order to evaluate the effectiveness of our proposed method under different variations (see Equation (3)), we evaluate $\sigma$ from 0.1 to 0.6 with the three-layer network. The result is shown in Figure 7. Without our proposed method, the accuracy degrades lower than $90 \%$ with $\sigma=0.2$. Our proposed method can maintain a high accuracy (95\%) with $\sigma=0.2$, and $90 \%$ accuracy with $\sigma=0.4$. Our proposed method represents one weight with one memristor while previous works $[5,9]$ use two memristors to represent one weight. As a result, we can't compare with them. The effectiveness of our proposed method is limited with high $\sigma$ in this experiment because the layer $256 \times 10$ has small number of rows. In next section, we evaluate the scalability of our proposed method. 


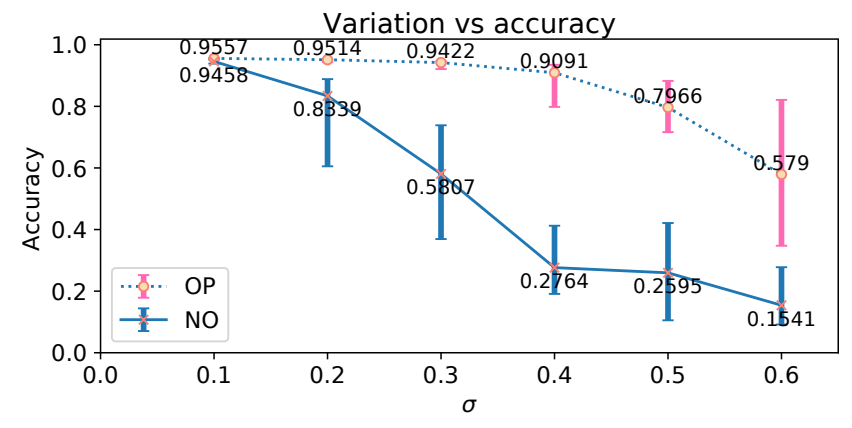

Figure 7: Accuracy vs $\sigma$ on the 3-layer network.

\subsection{Scalability}

As our method is sensitive to the size of crossbar $(S)$ and the number of rows of weight matrix $(R)$, our proposed method works when $S<R$. The effectiveness of our proposed method is sensitive to ratio $R / S$. In this section, we study the scalability of our method: how this ratio influences the effectiveness of our proposed method with the two layer network (see Table 1). We test different ratios with this network using two cases: 1) $20 \%$ SAF defect rate with variation $\sigma=0.4,2) 20 \%$ SAF defect rate with variation $\sigma=0.8$.

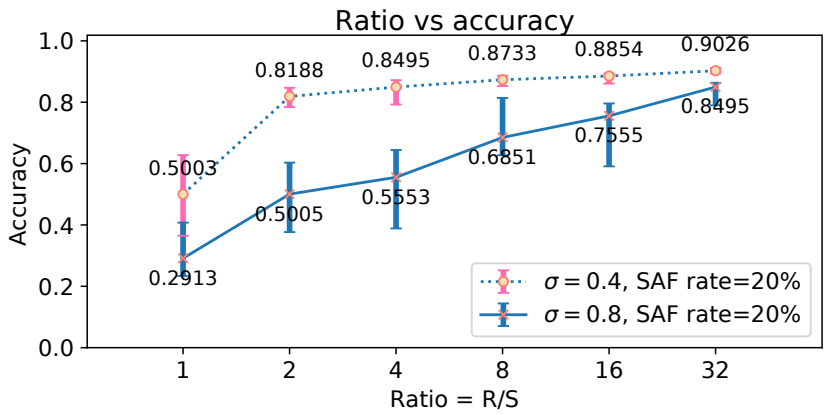

Figure 8: Accuracy vs ratio on the 1-layer network.

As shown in Figure 8, under large variation $\sigma=0.8$ with SAF defect, accuracy with our proposed method is increased as the ratio increases. Under small variation $(\sigma=0.4)$ with SAF defect, our proposed method can get a high accuracy $(81.88 \%$ ) when ratio $=2$ and the accuracy is also increased when the ratio increases.

\subsection{Discussion}

Our proposed method needs to rearrange inputs, which may lead to workload overhead for the controller and routers of the circuit. In our future work, we will optimize this phase in architecture level. Although we only test fully connected layers on the MNIST dataset, our proposed method should also works for larger networks on larger datasets as they also satisfies the phenomena which is observed in Section 3.2. We will experiment this in our future work.

Our proposed method needs an extra area to store the mapping solution in the system. Considering that tasks which are executed with edge devices should not be complicated enough, the networks should also not be complicated either. As a result, the extra storage is small. Taking one weight matrix with $1024 \times 1024$ as an example, the device only needs $1024 \times 1024 \times \log (1024)$ bit $<2 M B$ memory.

\section{CONCLUSION AND FUTURE WORK}

In this paper, we have discussed two reliability issues of MCAs for edge devices. Considering the limitation of MCA size, we have formulated the reliability problem as a $0-1$ programming problem based on analysis of SWV for edge devices. Based on the observation - different columns have similar weights, we have simplified the problem and proposed an optimal mapping method for the simplified problem. The experimental results have shown that the proposed method can recover $95 \%$ accuracy considering SAF defects and maintain a high accuracy $(90 \%)$ in variation $\sigma=0.4$. In our future work, we will optimize the whole computing system to execute our proposed method more effectively. Besides, we will evaluate the energy and area overhead.

\section{REFERENCES}

[1] G. Premsankar, M. Di Francesco, and T. Taleb, "Edge computing for the internet of things: A case study," IEEE Internet of Things fournal, vol. 5, no. 2, pp. 1275-1284, 2018.

[2] O. Krestinskaya, A. P. James, and L. O. Chua, "Neuromemristive circuits for edge computing: A review," IEEE Transactions on Neural Networks and Learning Systems, vol. 31, no. 1, pp. 4-23, 2020

[3] M. Hu et al., "Dot-product engine for neuromorphic computing: Programming $1 \mathrm{t} 1 \mathrm{~m}$ crossbar to accelerate matrix-vector multiplication," in DAC, 2016, pp. 1-6.

[4] Miao Hu et al., "Bsb training scheme implementation on memristor-based circuit," in IEEE Symposium on Computational Intelligence for Security and Defense Applications, 2013, pp. 80-87.

[5] B. Liu et al., "Vortex: Variation-aware training for memristor X-bar," in DAC, 2015, pp. 1-6.

[6] B. Liu et al., "Reduction and IR-drop compensations techniques for reliable neuromorphic computing systems," in ICCAD, 2014, pp. 63-70.

[7] C. Liu et al., "Rescuing memristor-based neuromorphic design with high defects," in $D A C, 2017$, pp. $1-6$.

[8] B. Zhang et al., "Handling stuck-at-faults in memristor crossbar arrays using matrix transformations." Association for Computing Machinery, 2019.

[9] L. Chen et al., "Accelerator-friendly neural-network training: Learning variations and defects in RRAM crossbar," in DATE, 2017, pp. 19-24.

[10] S. Jin, S. Pei, and Y. Wang, "A variation tolerant scheme for memristor crossbar based neural network designs via two-phase weight mapping and memristor programming," Future Generation Computer Systems, vol. 106, pp. 270 - 276, 2020.

[11] L. Xia et al., "Stuck-at fault tolerance in RRAM computing systems," IEEE fournal on Emerging and Selected Topics in Circuits and Systems, vol. 8, no. 1, pp. 102-115, 2018.

[12] B. Zhang et al., "Handling stuck-at-fault defects using matrix transformation for robust inference of dnns," TCAD, pp. 1-1, 2019.

[13] Q. Yang, H. Li, and Q. Wu, "A quantized training method to enhance accuracy of ReRAM-based neuromorphic systems," in ISCAS, 2018, pp. 1-5.

[14] A. Amirsoleimani et al., "In-Memory Vector-Matrix Multiplication in Monolithic Complementary Metal-Oxide-Semiconductor-Memristor Integrated Circuits: Design Choices, Challenges, and Perspectives," Advanced Intelligent Systems, p. 2000115, 2020 .

[15] M. Shevgoor et al., "Improving memristor memory with sneak current sharing," in ICCD, 2015, pp. 549-556.

[16] Y. Chen et al., "A survey of accelerator architectures for deep neural networks," Engineering, vol. 6, no. 3, pp. $264-274,2020$.

[17] Q. Xu et al., "Fault tolerance in memristive crossbar-based neuromorphic computing systems," Integration, vol. 70, pp. $70-79,2020$.

[18] S. Zhang et al., "Aging-aware lifetime enhancement for memristor-based neuromorphic computing," in DATE, 2019, pp. 1751-1756.

[19] L. Can et al., "Analogue signal and image processing with large memristor crossbars," Nature Electronics, pp. 52 - 59, 2018.

[20] S. R. Lee et al., "Multi-level switching of triple-layered taox RRAM with excellent reliability for storage class memory," in VLSIT, 2012, pp. 71-72.

[21] A. Shafiee et al., "ISAAC: A convolutional neural network accelerator with in-situ analog arithmetic in crossbars," in ISCA, 2016, pp. 14-26.

[22] M. A. Zidan et al., "Memristor-based memory: The sneak paths problem and solutions," Microelectronics fournal, vol. 44, no. 2, pp. 176 - 183, 2013.

[23] L. Yann, C. Corinna, and C. J. Burges, "The mnist database of handwritten digits," [Online], http://yann.lecun.com/exdb/mnist. 